RESEARCH ARTICLE

\title{
$1178 \mathrm{~J}, 527 \mathrm{~nm}$ near diffraction limited laser based on a complete closed-loop adaptive optics controlled off-axis multi-pass amplification laser system
}

\author{
Deen Wang ${ }^{1,2,3}$, Xin Zhang ${ }^{3}$, Wanjun Dai ${ }^{3}$, Ying Yang ${ }^{3}$, Xuewei Deng $^{3}$, Lin Chen ${ }^{1,2,3}$, Xudong Xie ${ }^{3}$, \\ Dongxia $\mathrm{Hu}^{3}$, Feng Jing ${ }^{3}$, Zeping Yang ${ }^{4}$, Qiang Yuan ${ }^{3}, \mathrm{Xiaofeng}_{\mathrm{Wei}^{3}}$, Qihua Zhu ${ }^{3}$, Wanguo Zheng ${ }^{3}$, \\ Xiaomin Zhang $^{3}$, and Lei Huang ${ }^{1,2}$ \\ ${ }^{1}$ Key Laboratory of Photonic Control Technology (Tsinghua University), Ministry of Education, Beijing 100084, China \\ ${ }^{2}$ State Key Laboratory of Precision Measurement Technology and Instruments, Department of Precision Instrument, \\ Tsinghua University, Beijing 100084, China \\ ${ }^{3}$ Research Center of Laser Fusion, CAEP, Mianyang 621900, China \\ ${ }^{4}$ Institute of Optics and Electronics, Chinese Academy of Sciences, Chengdu 610209, China \\ (Received 13 June 2020; revised 30 November 2020; accepted 19 January 2021)
}

\begin{abstract}
A $1178 \mathrm{~J}$ near diffraction limited $527 \mathrm{~nm}$ laser is realized in a complete closed-loop adaptive optics (AO) controlled off-axis multi-pass amplification laser system. Generated from a fiber laser and amplified by the pre-amplifier and the main amplifier, a $1053 \mathrm{~nm}$ laser beam with the energy of $1900 \mathrm{~J}$ is obtained and converted into a $527 \mathrm{~nm}$ laser beam by a KDP crystal with $62 \%$ conversion efficiency, $1178 \mathrm{~J}$ and beam quality of 7.93 times the diffraction limit (DL). By using a complete closed-loop AO configuration, the static and dynamic wavefront distortions of the laser system are measured and compensated. After correction, the diameter of the circle enclosing $80 \%$ energy is improved remarkably from 7.93DL to $1.29 \mathrm{DL}$. The focal spot is highly concentrated and the $1178 \mathrm{~J}, 527 \mathrm{~nm}$ near diffraction limited laser is achieved.
\end{abstract}

Keywords: kilojoule; near diffraction limit; 527 nm; X-ray

\section{Introduction}

X-ray imaging is an essential method to measure mass distribution and opacity of matter in high-energy density physics (HEDP) experiments, such as inertial confinement fusion $(\mathrm{ICF})^{[1,2]}$, magnetized liner inertial fusion $(\mathrm{MagLIF})^{[3,4]}$, z-pinch physics ${ }^{[5,6]}$, Thomson scattering ${ }^{[7]}$, and dynamic material properties researches ${ }^{[8]}$. High-energy lasers have become one of the most important tools ${ }^{[9-12]}$ to generate $\mathrm{X}$-rays with high spatial and temporal resolution ${ }^{[13]}$. When a high-energy laser beam is focused on a target, a hightemperature plasma is formed near the target surface and an X-ray is emitted from the plasma. In general, the performance of the generated X-ray is determined by

Correspondence to: L. Huang, Key Laboratory of Photonic Control Technology (Tsinghua University), Ministry of Education, Beijing 100084, China. Email: hl@tsinghua.edu.cn the characteristics of the laser, including output energy, wavelength, pulse length, and beam quality ${ }^{[14]}$. Compared with a $1 \mu \mathrm{m}$ laser beam, a $527 \mathrm{~nm}$ laser beam can reduce the risk of target back-reflection and improve the coupling efficiency of the X-ray, meanwhile avoiding serious absorption and optical damage when using the ultraviolet laser ${ }^{[15-18]}$. In particular, the X-ray with energy of several kiloelectronvolts, which is suitable for X-ray radiography in the HEDP experiments ${ }^{[4,6,16]}$, Thomson scattering, and compressed materials studies ${ }^{[7,8]}$, could be generated by using a kilojoule-class nanosecond-level $527 \mathrm{~nm}$ laser.

In these applications, the $527 \mathrm{~nm}$ laser beam should simultaneously possess kilojoule-level energy and high beam quality to generate the desired X-ray. To achieve high-energy output, a laser system with complex optical structure (e.g., multi-pass amplifier structure), numerous optical mirrors (generally more than 50 pieces), and large beam aperture (generally larger than $200 \mathrm{~mm}$ ) should be designed and built. 
However, wavefront distortion caused by imperfect installations and thermal effects of large-aperture optical components has become inevitable in such complex multi-pass amplification high-energy laser systems ${ }^{[19-24]}$. It seriously reduces the energy coupling efficiency and degrades the output laser beam quality. In other words, it is very hard for the $527 \mathrm{~nm}$ laser to achieve near diffraction limited (diffraction limit (DL), the size of the first zero of an Airy disk) beam quality and kilojoule-level energy simultaneously. Adaptive optics (AO) is the most commonly employed technology to correct the wavefront distortion in high-energy laser systems, including the National Ignition Facility in the USA and the Laser MégaJoule facility in France. For traditional AO technology, the deformable mirror (DM) is usually set as the cavity mirror of the multi-pass amplifier and in the middle of the beam path through it, while the Shack-Hartmann (SH) wavefront sensor is usually set in the output sensor package of the main-amplifier to ensure high efficiency correction on the wavefront distortion caused by thermal effects in the main amplifier ${ }^{[25-27]}$. However, the wavefront distortion generated from the output of the main amplifier to the target could not be corrected and still remains in the final laser beam, which will eventually have an influence on the intensity distribution of the focused target point ${ }^{[28]}$.

This paper presents a $1178 \mathrm{~J}$ near DL $527 \mathrm{~nm}$ laser using off-axis multi-pass amplification and complete closed-loop $\mathrm{AO}$. The $1053 \mathrm{~nm}$ laser beam generated from a fiber laser is amplified to $200 \mathrm{~mJ}$ by a rod-type pre-amplifier and then to $1900 \mathrm{~J}$ in the off-axis four-pass main amplifier. The $1053 \mathrm{~nm}$ laser beam is converted into a $527 \mathrm{~nm}$ laser beam with an energy of $1178 \mathrm{~J}$ by the $90^{\circ}$-cut type-I KDP crystal before being focused on the target. A complete closed-loop AO system consisting of a DM and an SH wavefront sensor is adopted in this laser. The DM is set after the main amplifier and an SH wavefront sensor is located near the target to measure the distorted wavefront transmitted by a beamsplitter and a collimating lens. The wavefront correction performance of the complete closed-loop AO configuration is simulated, and the results show that, by using the configuration, a $527 \mathrm{~nm}$ laser with near DL beam quality (1.12DL) could be achieved in the off-axis multi-pass amplification laser system. Finally, a $1178 \mathrm{~J}$ high-energy and 1.29DL highbeam-quality $527 \mathrm{~nm}$ laser is obtained and the intensity of the focal spot is highly concentrated at the target in the experiment. To the best of the authors' knowledge, this is the best quality laser among the $527 \mathrm{~nm}$, kilojoule-level class lasers.

\section{Design and fabrication of the laser system}

Figure 1 shows a schematic diagram of the $1178 \mathrm{~J}$ near diffraction limited $527 \mathrm{~nm}$ laser system using off-axis multipass amplification, which consists of seven primary parts, i.e., the front-end, the pre-amplifier, the main amplifier, the spatial filter, the reverser, the second harmonic generation (SHG), and the target. In the front-end part, a $1053 \mathrm{~nm}$ laser beam $(15 \mu \mathrm{J}, 1 \mathrm{~ns})$ generated from a fiber laser is collimated by lens $\mathrm{L} 1$ and then shaped into a $10 \mathrm{~mm} \times 10 \mathrm{~mm}$ superGaussian beam (17th order, as shown in Figure 1) by a square aperture (SA) liquid crystal amplitude modulator,

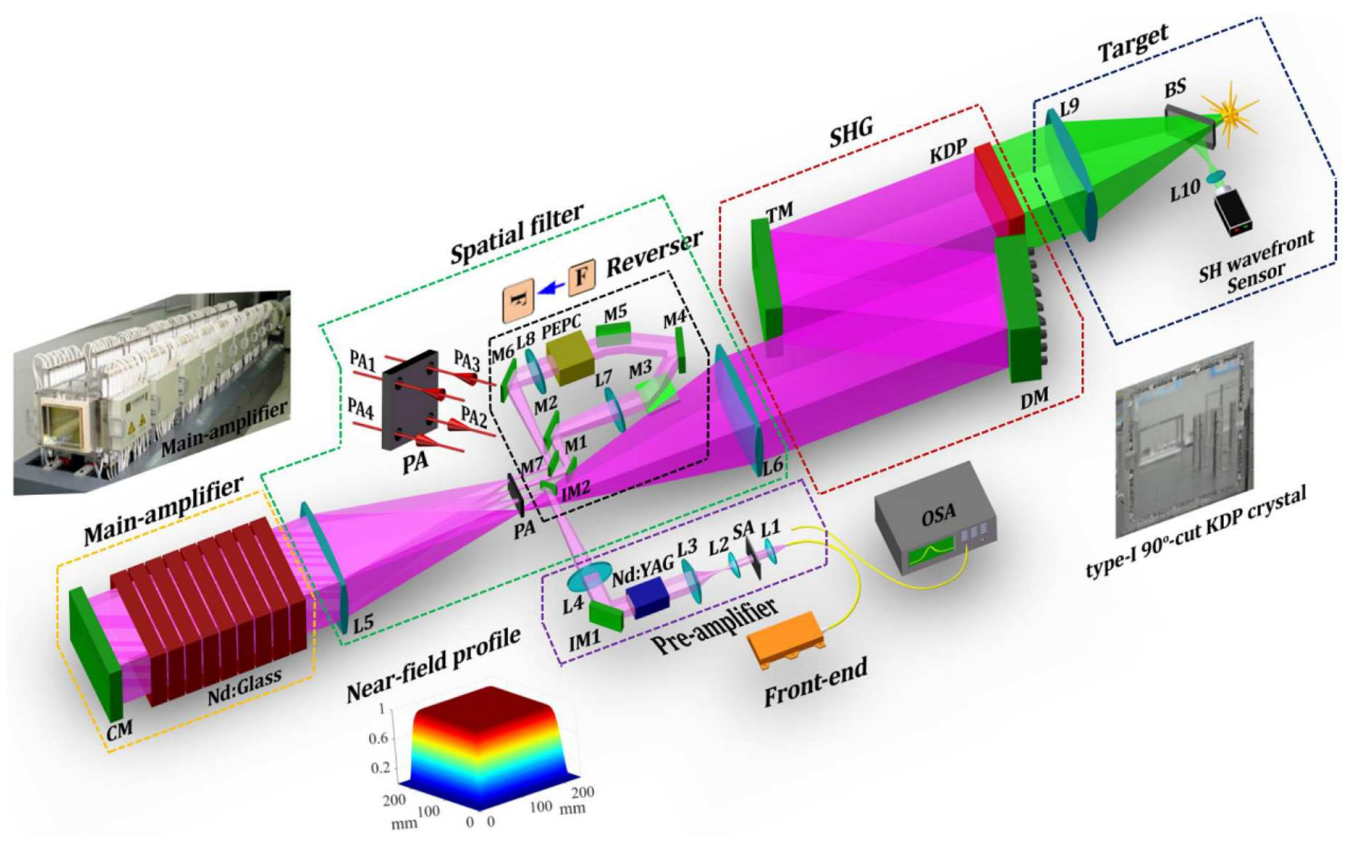

Figure 1. Schematic diagram of the $1178 \mathrm{~J}$ near diffraction limited $527 \mathrm{~nm}$ laser system using off-axis multi-pass amplification and complete closed-loop AO. BS, beamsplitter; CM, cavity mirror; DM, deformable mirror; L1-L10, focus lenses; M1-M7, IM1 and IM2, reflection mirrors; OSA, optical spectrum analyzer; PA, pinhole array; PEPC, plasma-electrode Pockels cell; SA, square aperture; TM, transport mirror. 
Table 1. Parameters of the lenses.

\begin{tabular}{lcccccccccc}
\hline Lens & L1 & L2 & L3 & L4 & L5 & L6 & L7 & L8 & L9 \\
\hline Aperture $(\mathrm{mm})$ & $\phi 20$ & $\phi 20$ & $\phi 70$ & $\phi 80$ & $260 \times 260$ & $260 \times 260$ & $80 \times 80$ & $80 \times 80$ & $260 \times 260$ & $\phi 15$ \\
Thickness $(\mathrm{mm})$ & 3 & 3 & 8 & 8 & 30 & 30 & 10 & 10 & 30 & 2 \\
Focal length $(\mathrm{m})$ & 0.15 & 0.15 & 0.69 & 1.38 & 6 & 6 & 2.1 & 1.75 & 2 \\
\hline
\end{tabular}

Table 2. Parameters of the reflection mirrors.

\begin{tabular}{lccccccc}
\hline Mirror & IM1 & IM2 & CM & M1, M7 & M2, M3, M4, M5, M6 & TM & BS \\
\hline Aperture (mm) & $60 \times 70$ & $15 \times 25$ & $260 \times 260$ & $45 \times 55$ & $80 \times 110$ & $260 \times 260$ & $50 \times 70$ \\
Thickness (mm) & 10 & 5 & 40 & 10 & 20 & 50 \\
\hline
\end{tabular}

which is commonly used for the laser beam shaping in highpower laser systems ${ }^{[29,30]}$. After being expanded to the size of $46 \mathrm{~mm} \times 46 \mathrm{~mm}$ by lenses L2 and L3, the laser beam enters into the pre-amplifier and is amplified to $200 \mathrm{~mJ}$ by an eight-pass Nd:YAG rod amplifier.

A spatial filter part, consisting of lenses L5, L6 and a pinhole array (PA), is set to achieve high spatial components filtering and laser beam collimation. After exiting from the pre-amplifier, the laser beam is reflected by reflection mirror IM1 and then converged by lens L4. After being reflected by reflection mirror IM2, the laser beam is injected into PA1 (i.e., the first filter pinhole in PA) and collimated to a $200 \mathrm{~mm}$ $\times 200 \mathrm{~mm}$ aperture beam by lens L5. Then, the beam enters into the main amplifier, which is composed of nine pieces of Nd:glass (410 $\mathrm{mm} \times 260 \mathrm{~mm}$, set at the Brewster angle). Reflected by the cavity mirror (CM), the laser beam extracts about $70 \mathrm{~J}$ energy from the main amplifier after passing through it twice and propagates back into PA2 (i.e., the second filter pinhole in PA). A high damage threshold pickoff mirror M1 is located behind PA2 to reflect the laser beam coming out of the spatial filter into the reverser, which is mainly composed of seven pieces of reflection mirrors (i.e., M1 to M7, high reflectivity for $1053 \mathrm{~nm}$ laser beam), two collimating lenses (i.e., L7 and L8), and a plasmaelectrode Pockels cell (PEPC). In the reverser part, the beam polarization and the near field of the beam are rotated $90^{\circ}$ by the PEPC and by three reflection mirrors (i.e., M3, M4, and M5), respectively. The combined use of the PEPC and the reflection mirrors could effectively isolate the self-excitation laser beam and mitigate the gain nonuniformity caused by the main amplifier. The primary parameters of these optical mirrors are listed in Tables 1 and 2. The diameters of spatial filter pinholes PA1 and PA2 are both 30DL, while those of PA1 and PA2 are both 40DL, as listed in Table 3. Here, as the laser beam in the system is in the square aperture, the DL is defined as ${ }^{[31]}$

$$
1 \mathrm{DL}=2 \lambda f / D
$$

where $\lambda$ is the wavelength of the laser beam, $f$ is the focal length of the lens, and $D$ is the side length of the square beam. It should be noted that the laser beam is focused by L4,
Table 3. Parameters of the spatial filter pinholes.

\begin{tabular}{lcccc}
\hline Pinhole & PA1 & PA2 & PA3 & PA4 \\
\hline Size (DL) & 30 & 30 & 40 & 40 \\
Size (mm) & $\phi 1.90$ & $\phi 1.90$ & $\phi 2.11$ & $\phi 2.11$ \\
\hline
\end{tabular}

L5, L7, and L5 (Figure 1) for the focal spots in the pinholes PA1, PA2, PA3, and PA4, respectively.

After passing through the reverser, the laser beam is emitted back into PA3 (i.e., the third filter pinhole in PA) by M7 and collimated into a $240 \mathrm{~mm} \times 240 \mathrm{~mm}$ aperture laser beam by lens $\mathrm{L} 5$ before entering into the main amplifier again. It should be noted that this time, the laser beam aperture is different from the first time (i.e., $200 \mathrm{~mm} \times$ $200 \mathrm{~mm}$ ). After passing through the main amplifier for third and fourth times, the laser beam extracts about 980 and $850 \mathrm{~J}$ of energy, respectively, and finally reaches as high as $1900 \mathrm{~J}$. The outputted $1053 \mathrm{~nm}$ laser beam is then collimated into a $240 \mathrm{~mm} \times 240 \mathrm{~mm}$ aperture beam by lens L6, after going through the fourth filter pinhole of PA (PA4). Then, reflected by the transport reflectors (at $7.5^{\circ}$ incident angle), including the DM and the transport mirror (TM), the $1053 \mathrm{~nm}$ laser beam is injected into a KDP crystal $(260 \mathrm{~mm} \times 260 \mathrm{~mm}$, $10 \mathrm{~mm}$ thickness, type $\mathrm{I}, 90^{\circ}$ cut) and converted into a $527 \mathrm{~nm}$ laser beam. The conversion efficiency is about $62 \%$ and the final $527 \mathrm{~nm}$ laser beam could reach as high as $1178 \mathrm{~J}$.

As discussed previously, in this off-axis multi-pass amplification laser system, energy is mainly provided by the largeaperture gain media (i.e., Nd:glass) in the main amplifier, and the off-axis amplification configuration ensures that the laser beam passes through the main amplifier four times to achieve high-energy extraction efficiency. The far-field image of the $1178 \mathrm{~J}, 527 \mathrm{~nm}$ laser is as poor as 7.93DL (diameter of the circle enclosing $80 \%$ energy) before wavefront correction (Section 3.2).In the off-axis four-pass amplification laser system, wavefront distortions, mainly comprising static and dynamic distortion ${ }^{[32]}$, could not be avoided completely. The primary source of static distortion is from fabrication imperfections and mounting stress in the optical components, especially large-aperture optics (e.g., CM, Nd:glass, L5, L6, TM), whereas dynamic distortion arises from thermal effects 


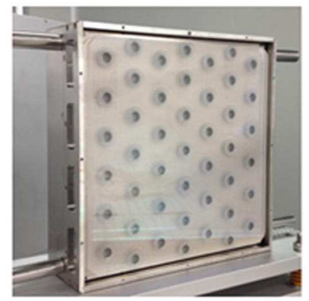

(a)

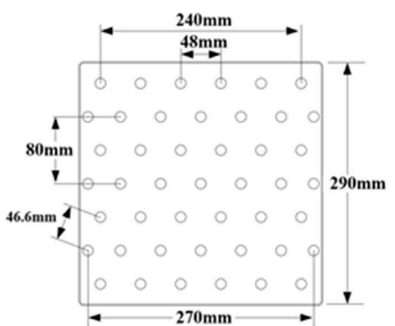

(c)

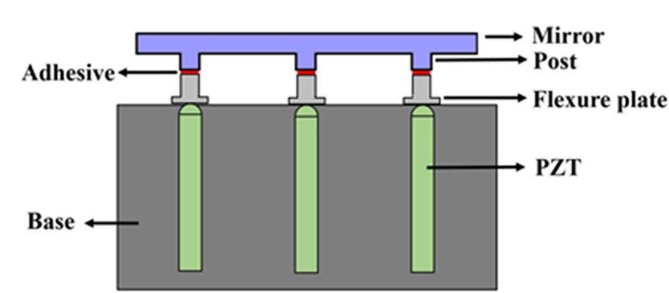

(b)

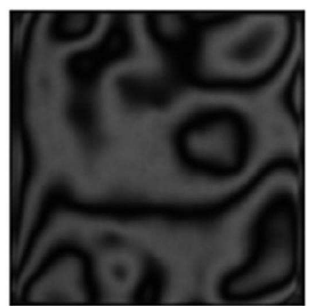

(d)

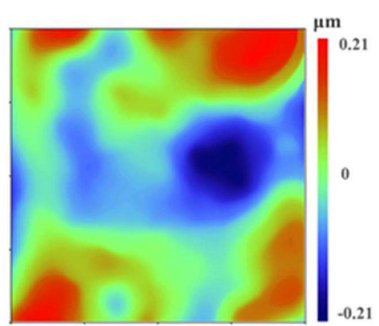

(e)

Figure 2. (a) Photo and (b) schematic diagram of the lab-manufactured DM; (c) hexagonally distributed 45 actuators in the DM; (d) interference fringe and (e) wavefront of the initial surface shape of the DM.

of the gain media in the main amplifier. Under the influence of the wavefront distortions, the beam quality degrades seriously and the brightness is limited (Figure 2), although the laser beam energy is high.

To improve the performance of the high-energy laser system, a complete closed-loop AO technology is used for the wavefront control from the front end to the target. As shown in Figure 1, in the complete closed-loop AO structure, an $\mathrm{SH}$ wavefront sensor composed of a $20 \times 20$ lenslet (the pitch is $250 \mu \mathrm{m}$ and the focal length is $7.5 \mathrm{~mm}$ ) and a CCD (Basler piA2400-17gm GigE, the pixel size is $3.45 \mu \mathrm{m} \times 3.45 \mu \mathrm{m}$ ) is located near the target to measure the distorted wavefront transmitted by a beamsplitter (BS; i.e., fused silica) and a focus lens (i.e., L10, biconvex lens, fused silica). The $527 \mathrm{~nm}$ laser beam is reflected by the BS and collimated by lens L10 before entering into the $\mathrm{SH}$ wavefront sensor. The BS is well polished and coated, and the peak-to-valley (PV) value and root-mean-square (RMS) value of the surface shape are $0.035 \mu \mathrm{m}$ and $0.0067 \mu \mathrm{m}$, respectively. The BS, L10, and $\mathrm{SH}$ wavefront sensor are mounted together on a mechanical base and the distortions introduced by these optical elements are calibrated before use in the experiment. After calibration, the measured distortion will be saved and regarded as system aberration. Before operation of the kilojoule-level high-energy laser, the mechanical base is moved out of the beamline to protect the BS, lens L10, and the SH wavefront sensor from being damaged by the high-energy laser beam. A lab-manufactured DM is located after the main amplifier to provide distortion correction and beam quality improvement. In the laser system, as the parallel laser beam is focused by lens L9 and then collimated by lens L10, the distance between lenses L9 and L10 is set as $2.05 \mathrm{~m}$ (i.e., equal to the sum of focal lengths of lenses L9 and L10). The distance between the DM and lens L9 is set as $2 \mathrm{~m}$ (i.e., equal to
Table 4. Key parameters of the lab-manufactured DM.

\begin{tabular}{lcccc}
\hline Parameters & Mirror & Post & Actuator & Base \\
\hline Material & BK7 & BK7 & $\begin{array}{c}\text { Piezoelectric } \\
\text { ceramic }\end{array}$ & Steel \\
Size & $290 \mathrm{~mm} \times$ & $\phi 10 \mathrm{~mm} \times$ & $7 \mathrm{~mm} \times$ & $320 \mathrm{~mm} \times$ \\
& $290 \mathrm{~mm} \times$ & $10 \mathrm{~mm}$ & $7 \mathrm{~mm} \times$ & $320 \mathrm{~mm} \times$ \\
& $10 \mathrm{~mm}$ & & $36 \mathrm{~mm}$ & $80 \mathrm{~mm}$ \\
\hline
\end{tabular}

the focal length of lens L9), and distance between the $\mathrm{SH}$ wavefront sensor and lens L10 is set as $50 \mathrm{~mm}$ (i.e., equal to the focal length of lens L10). Thus, the SH wavefront sensor is optically conjugated to the DM.

Figure 2(a) displays the lab-manufactured stacked array piezoelectric DM (mirror aperture $290 \mathrm{~mm} \times 290 \mathrm{~mm}$ ), which is specially designed and fabricated for the off-axis multi-pass amplification laser system. As shown in Figure 2(b), the DM consists of a BK7 mirror with 45 mirror posts, 45 hexagonally distributed piezoelectric ceramic transducer actuators (P887.91, driving voltage range of -20 to $120 \mathrm{~V}$, Physik Instrumente $\mathrm{GmbH}$ ), a flexure plate array and a steel base. As shown in Figure 2(c), the interval between each actuator in the DM is set as $48 \mathrm{~mm}$ along the horizontal direction, $40 \mathrm{~mm}$ along the vertical direction, and $46.6 \mathrm{~mm}$ along the diagonal direction of the hexagon. The mirror of the DM is well polished and coated by the dielectric film (hafnium oxide, $\mathrm{HfO}_{2}$ ) with high reflection (99.95\%) and high damage threshold $\left(9 \mathrm{~J} / \mathrm{cm}^{2}\right)$ at $1053 \mathrm{~nm}$ and $1 \mathrm{~ns}$ pulse duration. The interference fringe of the initial surface of assembled DM is shown in Figure 2(d) and the corresponding surface shape is displayed in Figure 2(e) with the PV value of $0.43 \mu \mathrm{m}$ and RMS value of $0.06 \mu \mathrm{m}$. The key parameters and performance features of the DM are listed in Tables 4 and 5. 


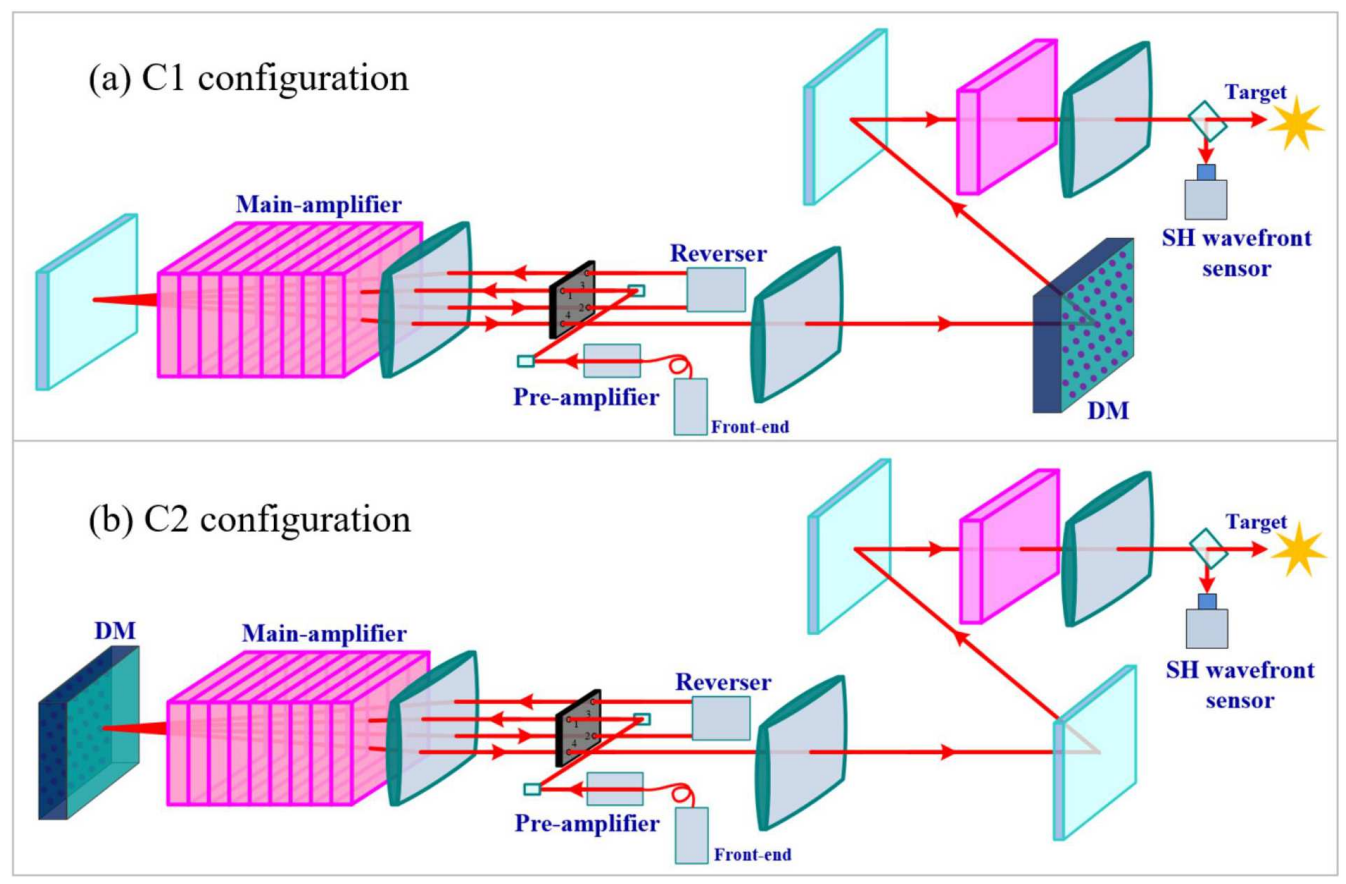

Figure 3. Optical schemes for configurations (a) $\mathrm{C} 1$ and (b) $\mathrm{C} 2$.

Table 5. Performance of the lab-manufactured DM.

\begin{tabular}{lc}
\hline Parameters & Value \\
\hline Damage threshold of the mirror & $9 \mathrm{~J} / \mathrm{cm}^{2} @ 1053 \mathrm{~nm}, 1 \mathrm{~ns}$ \\
Reflectivity of the mirror & $99.95 \% @ 1053 \mathrm{~nm}, 1 \mathrm{~ns}$ \\
Stroke of the actuator & $12 \mu \mathrm{m}$ \\
Nonlinearity of the actuator & $5 \%$ \\
Hysteresis of the actuator & $2 \%$ \\
Initial surface shape of the DM & $0.43 \mu \mathrm{m}(\mathrm{PV}), 0.06 \mu \mathrm{m}(\mathrm{RMS})$ \\
Open-loop bandwidth of the DM & $50 \mathrm{~Hz}$ \\
\hline
\end{tabular}

\section{Simulation and experiment}

\subsection{Simulation analysis}

To verify the wavefront distortion correction capability of the complete closed-loop AO, a numerical model was built and the simulation was implemented. As shown in Figure 3(a), the DM is set after the main amplifier in this AO configuration (denoted as configuration $\mathrm{C} 1$ in the simulation). Note that, in the simulation, the traditional AO configuration (denoted as configuration $\mathrm{C} 2$ ) of setting the DM at the end of the amplifier cavity is taken as a comparison (Figure 3(b)). For both configurations, wavefront distortion of the entire beamline is measured by the $\mathrm{SH}$ wavefront sensor located at the target and taken as the correction object.

In configuration $\mathrm{C} 1$, the laser beam is reflected by the DM once the incident angle is $7.5^{\circ}$ and the beam aperture on the DM is $240 \mathrm{~mm} \times 242 \mathrm{~mm}$ as shown in Figure 4(a). In configuration $\mathrm{C} 2$, the laser beam is reflected by the DM twice (Figure 4(c)). For the first reflection, the laser beam aperture on the DM is $200 \mathrm{~mm} \times 200 \mathrm{~mm}$. For the second reflection, the polarization and the near field of the beam are rotated $90^{\circ}$ and the laser beam aperture on the DM is $240 \mathrm{~mm} \times 240 \mathrm{~mm}$.

In the simulation, a finite element model of the DM is constructed in COMSOL Multiphysics software ${ }^{[33,34]}$ to obtain its influence function. In the finite element model, the DM consists of a BK7 mirror with 45 mirror posts, 45 hexagonally distributed actuators, a flexure plate array, and a steel base (Figure 2). Primary parameters of the DM are listed in Tables 4 and 5. The degree of freedom of the steel base and the hysteresis of the actuator are both set to zero. The initial surface shape of the mirror is set to be an ideal plane without any distortion. In the simulation, no external force is applied on the DM, and none of the thermal performance of the DM is taken into consideration, including heating of the base material, the glass substrate, and the actuators. By using the finite element model, the influence functions of DM could be obtained. Note that the target wavefront distortion in the simulation is calculated by using Zernike polynomials and measurement data instead of this finite element model. In the wavefront correction simulation, the sizes of pinholes are set to be infinite, as they have no influence on the wavefront correction procedure.

Owing to changes in the beam size and the beam rotation, the influence functions of the same actuator are different for configurations $\mathrm{C} 1$ and $\mathrm{C} 2$. Taking the actuator marked with a blue dashed square in Figure 4(a) as an example, its influence function in configuration $\mathrm{C} 1$ is a common Gaussian-type shape as shown in Figure 4(b), but the function in configuration $\mathrm{C} 2$ has two Gaussian-type peaks as the laser beam is reflected by the DM twice. In the influence function shown 


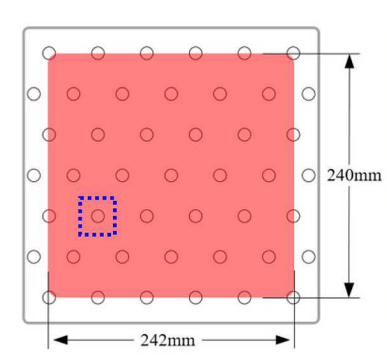

(a)

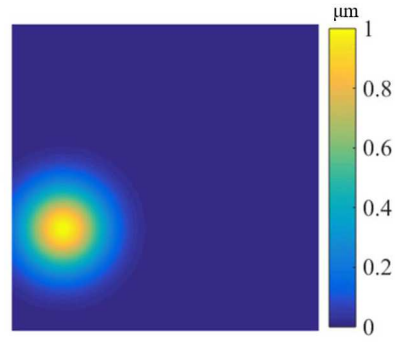

(b)

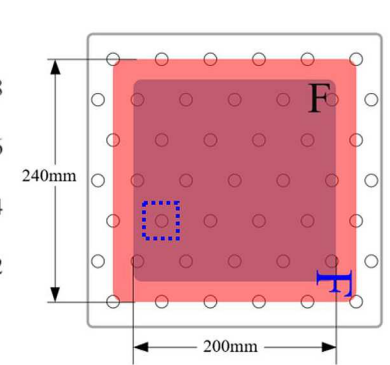

(c)

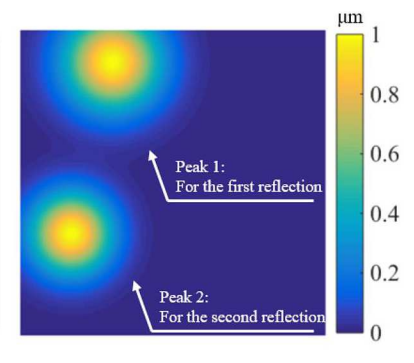

(d)

Figure 4. (a), (c) Laser beam on the DM in configurations (a) C1 and (c) C2. (b), (d) Influence functions for the same actuator (i.e., the actuator in the blue dashed square in (a) and (c)) for configurations (b) $\mathrm{C} 1$ and (d) $\mathrm{C} 2$.

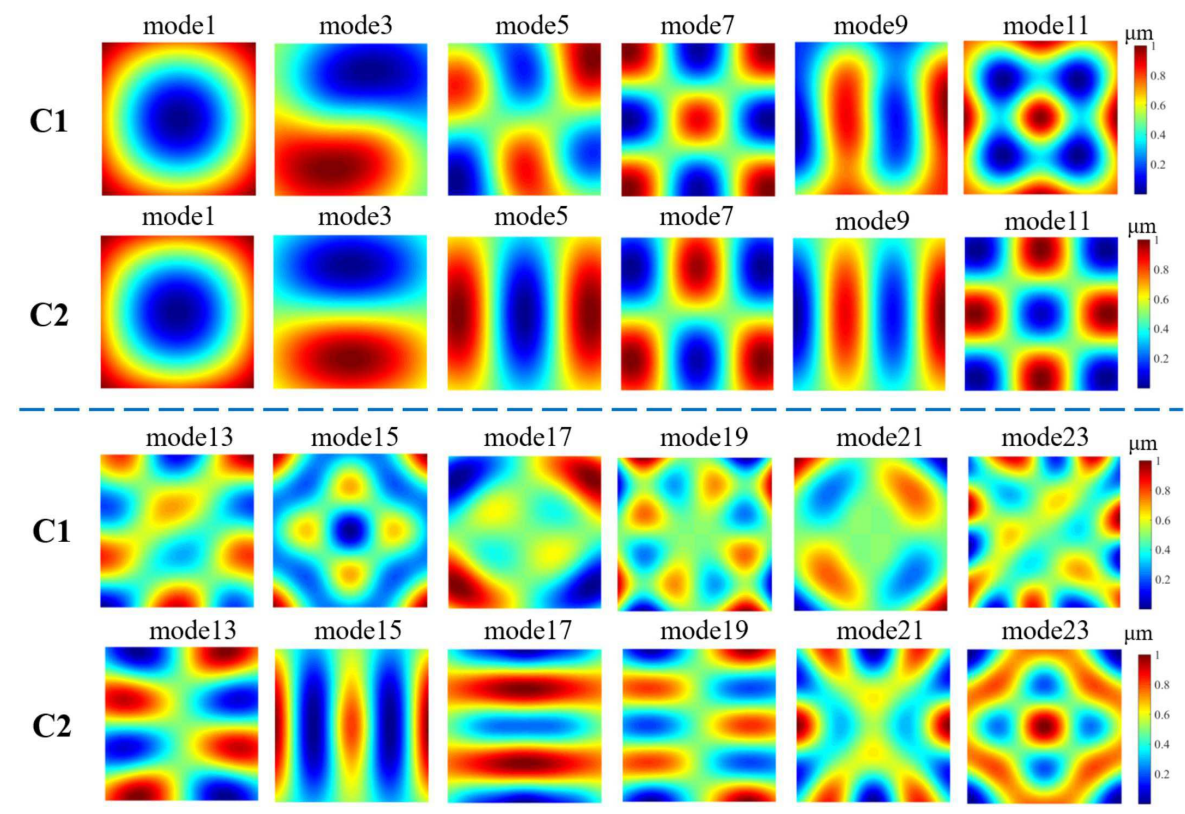

Figure 5. Comparison between the representative eigenmodes of configurations $\mathrm{C} 1$ and $\mathrm{C} 2$. The first and third rows are for configuration $\mathrm{C} 1$ and the second and fourth rows are for configuration $\mathrm{C} 2$.

in Figure 4(d), peak 1 is for the first reflected laser beam in Figure 4(c) and the same as that in Figure 4(b), whereas peak 2 is for the $90^{\circ}$ rotated laser beam in Figure 4(c). Based on the influence function, the crosstalk of the center actuator in configuration $\mathrm{C} 1$ is calculated to be 0.20 , whereas it is much more complex for configuration $\mathrm{C} 2$. Figure 5 shows the representative eigenmodes of $\mathrm{C} 1$ and $\mathrm{C} 2$ configurations, which represent the wavefront correction capability of the corresponding configuration, as the linear combinations of the eigenmodes are exactly the wavefront modes that could be achieved by the corresponding configuration ${ }^{[35]}$. However, the eigenmodes of a DM in practical application are somewhat different from the ideal modes displayed in Figure 5 owing to the limited stroke of the DM.

To compare the correction capability of configurations $\mathrm{C} 1$ and $\mathrm{C} 2$, the 3rd to 15th Zernike-mode aberrations are taken as the target wavefront distortions ${ }^{[36]}$ and the $\mathrm{PV}$ value of each aberration is set as $4 \mu \mathrm{m}$. Figure 6 shows the aberration correction results by using configurations $\mathrm{C} 1$ and
C2. For configuration $\mathrm{C} 1$, the $\mathrm{PV}$ values of the residual errors are all less than $0.2 \mu \mathrm{m}$ and the RMS values are less than $0.02 \mu \mathrm{m}$. Except for the 4th, 5th, 11th, and 12th Zernike modes (astigmatism-type aberrations, as shown in Figure 7), configuration $\mathrm{C} 2$ has similar correction capability to configuration $\mathrm{C} 1$. However, the correction capability of configuration $\mathrm{C} 2$ is weak and the residuals are significant (PV values are all larger than $0.4 \mu \mathrm{m}$ and RMS values are larger than $0.05 \mu \mathrm{m}$ ) for the 4th, 5th, 11th, and 12th Zernike modes. From the analysis above, configuration $\mathrm{C} 1$ excels over configuration $\mathrm{C} 2$ in correction capability for the typical 3rd to 15 th Zernike modes.

As is well known, in high-energy laser systems, when laser beam passes through the pinhole, large wavefront distortion will enlarge the focal spot in the pinhole, increase the plasma-closure risk (i.e., plasma could be generated when the metal material is ionized by the laser beam) and distinctly affect the transport efficiency ${ }^{[37,38]}$. Thus, the wavefront distortions of the laser beam passing through the pinholes PA2, 

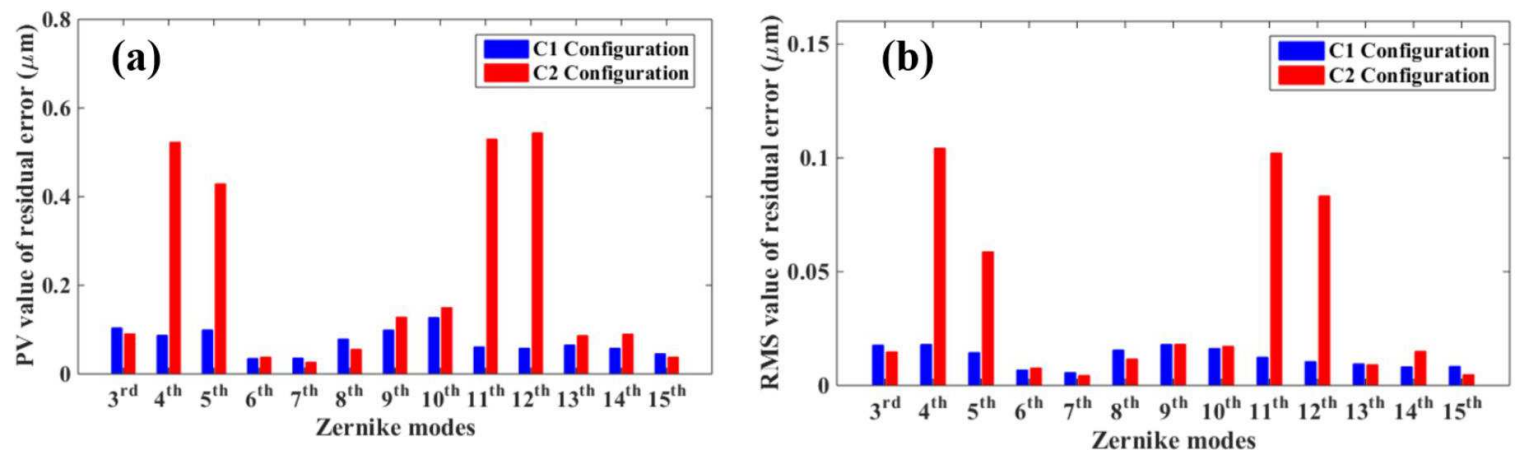

Figure 6. Correction results of configurations $\mathrm{C} 1$ and $\mathrm{C} 2$ for the 3rd to 15th Zernike-mode aberrations: (a) PV and (b) RMS values of the residual errors.
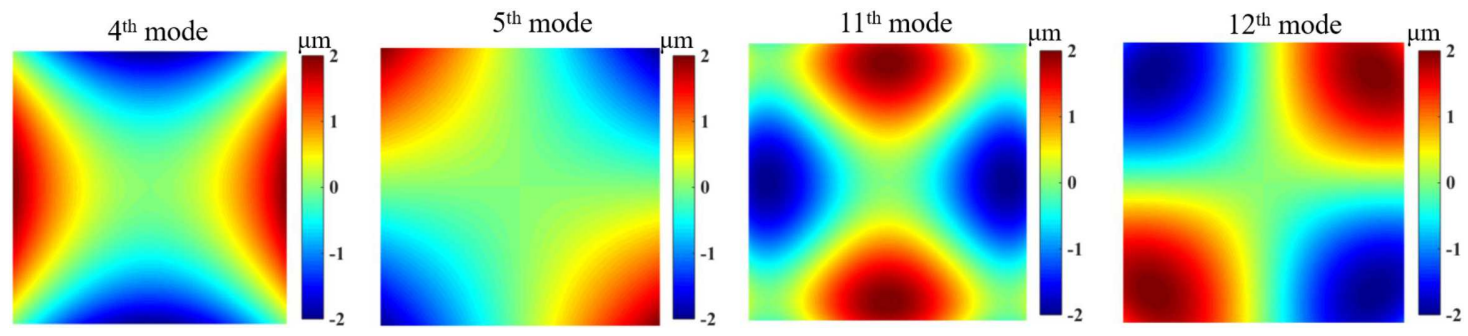

Figure 7. The 4th, 5th, 11th, and 12th Zernike modes.
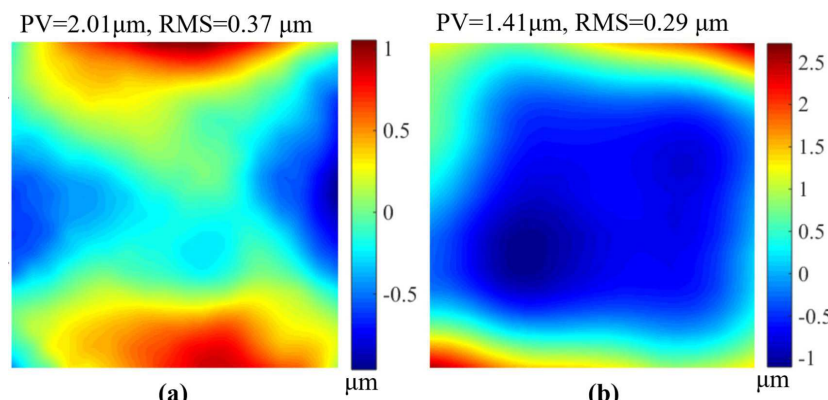

Figure 8. The target wavefront distortions to be corrected: (a) static wavefront distortion and (b) dynamic wavefront distortion.

PA3, and PA4 are taken into consideration in the simulation. The target static wavefront distortion is generated by random superposition of the 1st- to 15th-order Zernike polynomials, as shown in Figure 8(a). According to the reality of highenergy laser systems, the amplitude is set in the $\pm 2 \mu \mathrm{m}$ range for the 1st- to 5th-order polynomials, $\pm 1 \mu \mathrm{m}$ for the 6th- to 10th-order polynomials, and $\pm 0.5 \mu \mathrm{m}$ for the 11thto 15 th-order polynomials. The target dynamic wavefront distortions to be corrected in the simulation are generated according to the measurement data from the multi-pass laser system, as shown in Figure 8(b). Note that the target static and dynamic wavefront distortions of the entire beamline (shown in Figure 8) are the same for configurations $\mathrm{C} 1$ and $\mathrm{C} 2$ in the simulation.

According to the target wavefront distortions shown in Figure 8, Figures 9(a1)-9(d1) display the wavefront distortions of the laser beam to be corrected on the image plane before pinholes PA2, PA3, and PA4 and at the target before correction, consisting of the static and dynamic wavefront distortions with the PV values of 1.32, 1.41, 2.20, and $3.43 \mu \mathrm{m}$, respectively. By using the fast Fourier algorithm, the far-field intensity distribution can be calculated based on the wavefront distortions and the 17th-order super-Gaussian near-field profile. The corresponding distributions of the focal spots in the pinholes PA2, PA3, and PA4 and at the target are shown in Figures 9(a2)-9(d2), with 80\% energy concentrating within circles of diameters 2.61DL, 3.23DL, 5.54DL, and $8.89 \mathrm{DL}$, respectively. It should be noted that the laser beam is focused by L5, L8, L5, and L9, respectively (Figure 1) for the focal spots in the pinholes PA2, PA3, and PA4 and at the target.

When using $\mathrm{C} 1$ configuration, the DM is controlled to generate a new surface shape to compensate for the wavefront distortion of the entire beamline detected by the SH wavefront sensor. The least-squares algorithm is adopted to calculate the compensation surface shape of the DM in the simulation. Figure 10(a) shows the surface shape of the DM after correction, with the PV value of $1.93 \mu \mathrm{m}$ and the RMS value of $0.72 \mu \mathrm{m}$. The wavefront distortion of the $527 \mathrm{~nm}$ laser beam is well reduced, with the PV and RMS values of the residual error dropping to $0.37 \mu \mathrm{m}$ and $0.07 \mu \mathrm{m}$ (Figure $10(\mathrm{c})$ ), respectively. The diameter of the circle enclosing $80 \%$ energy of the focal spot is about 1.12DL (Figure 10(d)). As the DM is located after the main amplifier and spatial filter, the wavefront distortions of the laser beam in the pinholes PA2, PA3, and PA4 are unaffected by the DM and remain unchanged. 


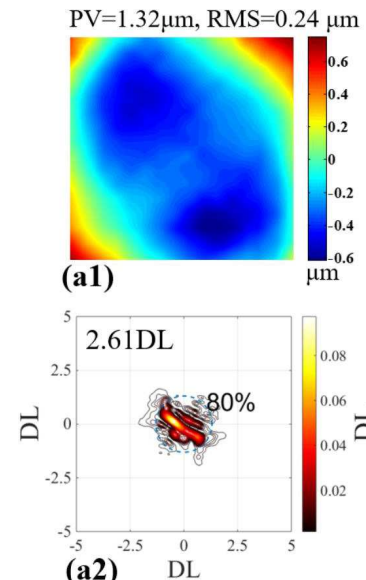

(a2) DL
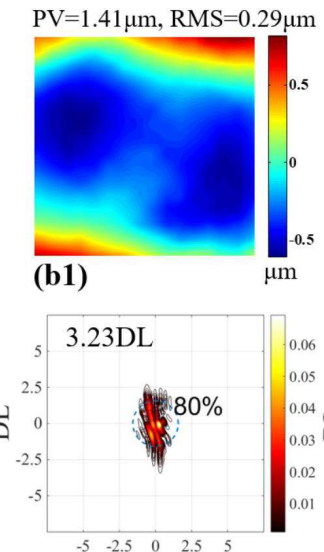

(b2) ${ }^{-2.5}$ DL
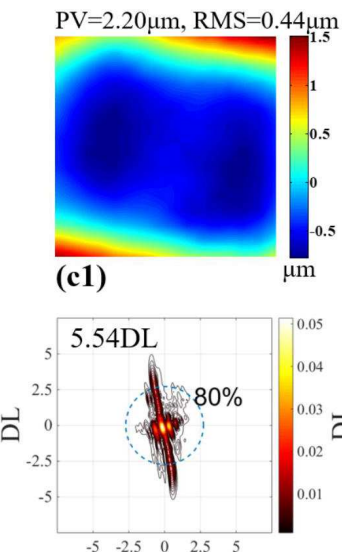

(c2) $\mathrm{DL}$
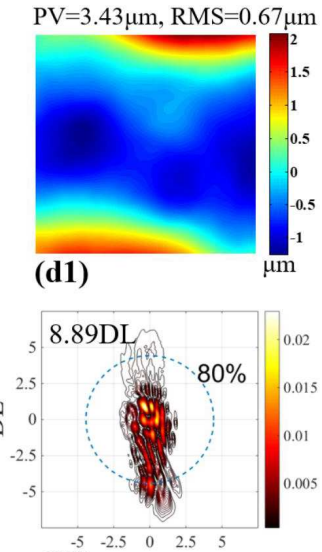

(d2) DL

Figure 9. The wavefront distortions of the laser beam on the image plane before pinholes (a1) PA2, (b1) PA3, and (c1) PA4 and (d1) at the target; (a2)-(d2) are the corresponding distributions of the focal spots.

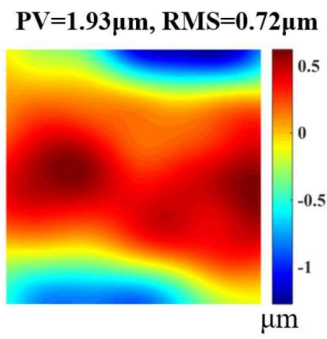

(a)

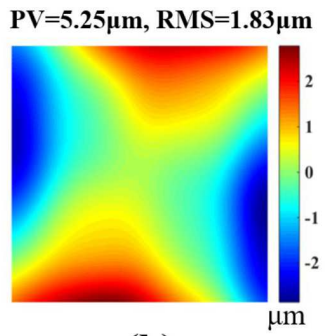

(b)

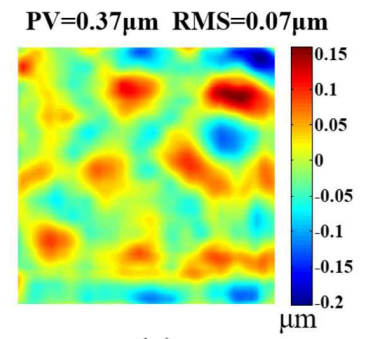

(c)

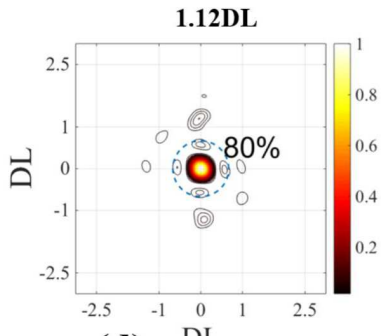

(d)

Figure 10. (a), (b) Generated surface shapes of the DM to compensate the wavefront distortions for configurations C1 and C2, respectively; (c) residual wavefront error at the target after correction by using configuration $\mathrm{C} 1$; (d) distribution of the target focal spot after correction by using configuration $\mathrm{C} 1$.

When using configuration $\mathrm{C} 2$, the DM is set as the cavity mirror of the main amplifier. As shown in Figure 10(b), when the DM is adjusted to compensate for the wavefront distortion of the entire beamline, the wavefront distortion of the laser beam passing through the main amplifier will be obviously influenced by the new surface shape of the DM (Figure 10(b); PV value of $5.25 \mu \mathrm{m}$ and RMS value of $1.83 \mu \mathrm{m}$ ). Similar to the correction result for configuration $\mathrm{C} 1$, the wavefront distortion of the $527 \mathrm{~nm}$ laser beam is well reduced with the PV value of $0.61 \mu \mathrm{m}$ and RMS value of $0.12 \mu \mathrm{m}$ (Figure 11(d1)) after correction. However, the wavefront distortions of the laser beam on the image plane before pinholes PA2, PA3, and PA4 grow worse. Figures 11(a1)-11(c1) demonstrate the wavefront distortions of the laser beam on the image plane before pinholes PA2, PA3, and PA4 with PV values of 7.02, 8.01, and $2.43 \mu \mathrm{m}$, respectively, after the wavefront distortion of the entire beamline is corrected. The distributions of the focal spots are shown in Figures 11(a2)-11(d2), corresponding to the wavefront shown in Figures 11(a1)-11(d1). As seen from Figure 11(b2), the intensity distribution of the focal spot in the pinhole PA3 is as poor as 13.83DL $(80 \%$ energy encircled) in diameter, which would bring serious plasma-closure risk and cause fatal damage to the optical components.
From the simulation results, the complete closed-loop AO configuration (i.e., configuration $\mathrm{C} 1$ ) has the unique advantage in the entire beamline wavefront distortion correction and beam quality improvement for the off-axis multi-pass amplification laser system.

\subsection{Experiment and discussion}

Based on the off-axis multi-pass amplification and the complete closed-loop AO configuration (i.e., configuration $\mathrm{C} 1$ ), a verification experiment is carried out and the $527 \mathrm{~nm}, 1 \mathrm{~ns}$, and $1178 \mathrm{~J}$ laser with near diffraction limited beam quality is achieved. In the experiment, before the $527 \mathrm{~nm}, 1178 \mathrm{~J}$ laser works, the static and dynamic wavefront distortions of the entire beamline are measured and then superposed together to form the to-be-corrected target distortion.

First, a $1053 \mathrm{~nm}$ front-end seed beam $(1 \mathrm{~Hz}, 15 \mu \mathrm{J}, 1 \mathrm{~ns})$, coming out from the front-end part and passing through the pre-amplifier and main amplifier, is converted into a $527 \mathrm{~nm}$ laser beam $(1 \mathrm{~Hz}, 5 \mathrm{~nJ}, 1 \mathrm{~ns})$ by the KDP crystal, whereas the pre-amplifier and main amplifier are on standby. The $527 \mathrm{~nm}$ beam is detected and the static wavefront distortion of the entire beamline is measured by the $\mathrm{SH}$ wavefront sensor at the target. Figure 12(a) shows the measured static 


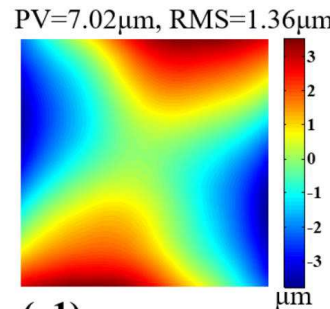

(a1)

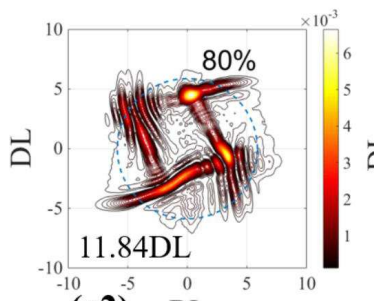

(a2) DL

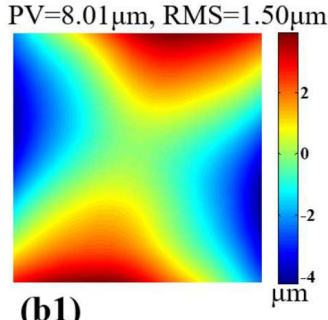

(b1)

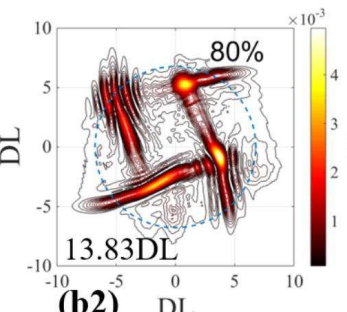

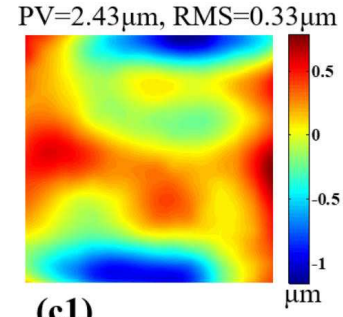

(c1)

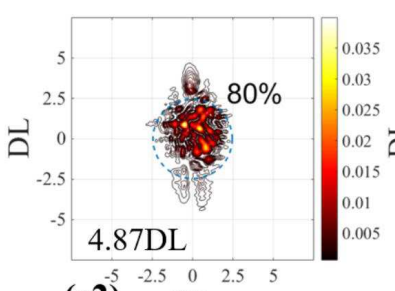

(c2) ${ }^{-5} \quad$ DL

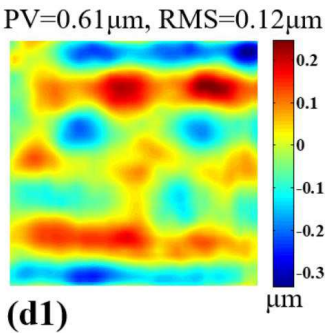

(d1)

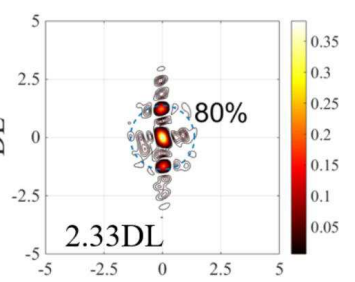

(d2)

Figure 11. Residual errors of the wavefront distortions on the image plane before pinholes (a1) PA2, (b1) PA3, and (c1) PA4 and (d1) at the target after correction by using the configuration $\mathrm{C} 2$; (a2)-(d2) corresponding distributions of the focal spots.

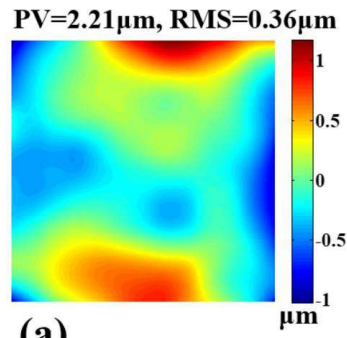

(a)

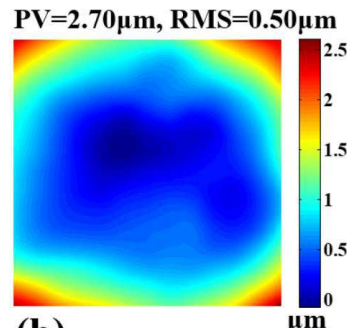

(b)

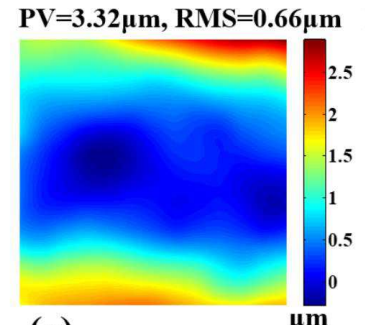

(c)

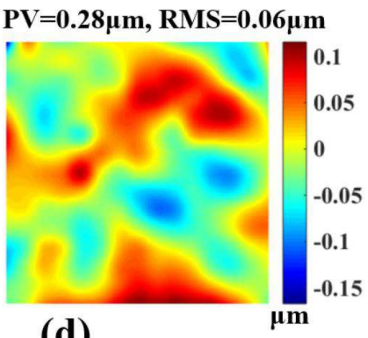

(d)

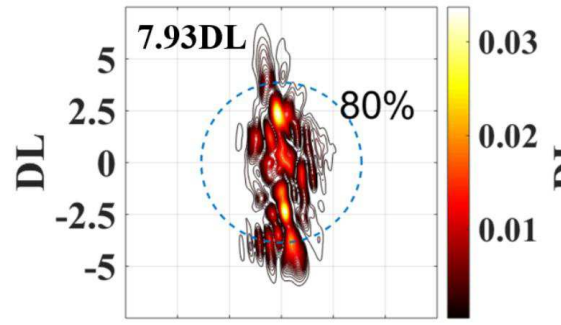

(e)
$-5-2.502 .55$

DL

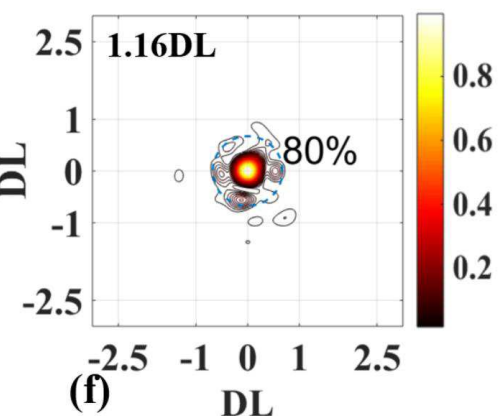

Figure 12. Experimental results: (a) static wavefront distortion of the entire beamline; (b) dynamic wavefront distortion of the main amplifier; (c) total wavefront distortion of the entire beamline composed of static and dynamic wavefront distortions; (d) residual error of the wavefront distortion at the target after correction; (e) distribution of the focal spot at the target before correction; (f) distribution of the focal spot at the target after correction.

wavefront distortion of the entire beamline with the PV value of $2.21 \mu \mathrm{m}$.

Second, the thermal distortion of the main amplifier is measured, which is taken as the primary dynamic distortion of the entire laser system. In the measurement, the flashlamps in the main amplifier are activated and the main amplifier is set to work. The $1053 \mathrm{~nm}$ front-end seed beam (single pulse, $1 \mu \mathrm{J}, 1 \mathrm{~ns}$ ) from the front-end part is amplified to about $1 \mathrm{~mJ}$ by the main amplifier and converted into about $5 \mu \mathrm{J}$ by the KDP crystal. By setting the acquired static wavefront distortion as the reference, the thermal distortion of the $\mathrm{Nd}$ :glass in the main amplifier is measured by the $\mathrm{SH}$ wavefront sensor. Note that a $0.1 \%$ attenuator (i.e., OD 3.0 filter, with OD referring to optical density) is added in front of the $\mathrm{SH}$ wavefront sensor to avoid laser beam damage in the dynamic distortion measurement. Figure 12(b) shows the measured dynamic distortion of the entire beamline with a PV value of $2.70 \mu \mathrm{m}$. Note that, during these two steps, the DM maintains its initial surface shape (i.e., Figures 2(d) and 2(e)) without any driving voltage exerted on it.

Third, taking the acquired static and dynamic wavefront distortions as the correction object, the entire beamline 

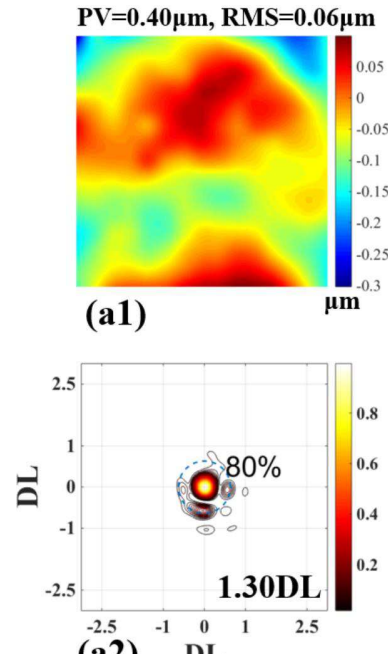

(a2) DL
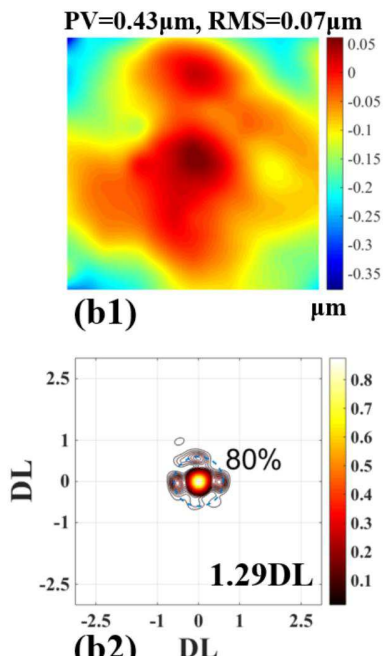
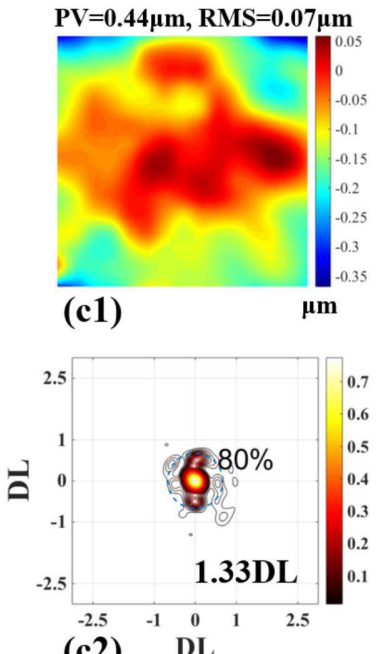

(c2)

Figure 13. Measured wavefront distortion of the $527 \mathrm{~nm}$ laser at the target and the corresponding distribution of the focal spot for: (a1), (a2) the first shot; (b1), (b2) the second shot; (c1), (c2) the third shot.

wavefront distortion is corrected by the DM and the final control voltage matrix of the actuators in the DM is calculated and stored. When the pre-amplifier and main amplifier cool down completely, the $1053 \mathrm{~nm}$ front-end seed beam ( $1 \mathrm{~Hz}, 15 \mu \mathrm{J}, 1 \mathrm{~ns}$ ) is emitted from the front-end part. After passing through the laser system, the $1053 \mathrm{~nm}$ laser beam is converted into a $527 \mathrm{~nm}$ laser beam by the KDP crystal and then gets into the $\mathrm{SH}$ wavefront sensor. By using the $1 \mathrm{~Hz} 527 \mathrm{~nm}$ front-end seed beam, the influence functions of the DM can be measured by the SH wavefront sensor and recorded. The total distortion of the entire beamline is obtained with a PV value of $3.32 \mu \mathrm{m}$ as shown in Figure 12(c) by superposing the static and dynamic wavefront distortions. Based on the total distortion and the influence functions of the DM, a least-squares algorithm is employed to calculate the driving voltage matrix of the actuators in the DM, whereas the residual error is taken as the judgment criterion. After five iterations, the PV value of the residual error converges to a stable value, thus the control loop being accomplished. Then, the final driving voltage matrix of the actuators in the DM is achieved and stored. Figure 12(d) displays the residual error of the corrected wavefront with a PV value of $0.28 \mu \mathrm{m}$ and RMS value of $0.06 \mu \mathrm{m}$. The distribution of the focal spot at the target before correction is shown in Figure 12(e), where the $x$-axis and $y$-axis are both scaled from $-7.5 \mathrm{DL}$ to $7.5 \mathrm{DL}$. The diameter of the circle enclosing $80 \%$ energy of the focal spot is $7.93 \mathrm{DL}$. The distribution of the focal spot at the target after correction is displayed in Figure 12(f), where the $x$-axis and $y$-axis are both scaled from -3DL to 3DL. After correction, the intensity of the focal spot is highly concentrated and the diameter of the circle enclosing $80 \%$ energy is as small as $1.16 \mathrm{DL}$.

Considering that the thermal distortion of the main amplifier could be regarded approximately as the same for other
Table 6. Residual errors and beam quality for three consecutive shots.

\begin{tabular}{lccc}
\hline Laser shot & 1st & 2nd & 3rd \\
\hline PV value of the residual error $(\mu \mathrm{m})$ & 0.40 & 0.43 & 0.44 \\
Diameter of circle enclosing $80 \%$ energy (DL) & 1.30 & 1.29 & 1.33 \\
\hline
\end{tabular}

laser shots, the wavefront distortion of the entire beamline could be taken as a constant value. Thus, the ultimate surface shape of the DM, which is aimed to achieve the distortion correction in the third step, could be used to realize precorrection for dynamic laser shots of this multi-pass amplification laser system. In the experiment, the driving voltages exerted on the DM are kept unchanged and correspondingly the surface shape of the DM remains stable during the laser operation. Figure 13 shows the measurement results for three consecutive laser shots. Note that the interval time between two adjacent shots is set as no less than 2 hours to ensure the main amplifier cools down completely. Figures 13(a1)13(c1) and 13(a2)-13(c2) show the measured wavefront distortions of the $527 \mathrm{~nm}$ laser beam at the target and the corresponding distributions of the focal spot for the first, second, and third shot, respectively. The PV values of the residual errors are $0.40,0.43$, and $0.44 \mu \mathrm{m}$ and the RMS values are $0.06,0.07$, and $0.07 \mu \mathrm{m}$, respectively, whereas the diameters of the circles enclosing $80 \%$ energy of the focal spots are 1.30DL, 1.29DL, and 1.33DL respectively, as listed in Table 6. It should be noted that the slight differences between the correction effects for the three laser shots result from the dynamic distortion varieties and the thermal residual. In the future, real-time measurement technique for thermal distortion and optimized correction algorithm would be considered to use for improving the beam quality further. 


\section{Conclusion}

In conclusion, a $1178 \mathrm{~J}$ near diffraction limited $527 \mathrm{~nm}$ laser has been achieved by using an off-axis multi-pass amplification structure composed of a front-end part, preamplifier, main amplifier, spatial filter, reverser, SHG, and target using a complete closed-loop AO configuration consisting of an SH wavefront sensor and a DM. In the laser system, the main amplifier is one of the most important parts providing more than $99.9 \%$ energy for the $1053 \mathrm{~nm}$ laser beam, which reaches as high as $1900 \mathrm{~J}$ after passing through the main amplifier four times. The $1053 \mathrm{~nm}$ laser beam is converted into a $527 \mathrm{~nm}$ laser beam by a KDP crystal with a conversion efficiency of $62 \%$, energy of $1178 \mathrm{~J}$, and beam quality of 7.93DL. A complete closed-loop AO configuration has been adopted to compensate for the wavefront distortion of the laser system and improve the beam quality of the laser beam by using a lab-manufactured DM and a leastsquares algorithm. After correction, the diameter of the circle enclosing $80 \%$ energy is $1.29 \mathrm{DL}$ and the intensity of the focal spot is highly concentrated. Thus, the beam quality of the $1178 \mathrm{~J}, 527 \mathrm{~nm}$ laser is improved up to near DL.

\section{Funding}

This work was supported by the National Natural Science Foundation of China (Nos. 61775199 and 61775112).

\section{Disclosure}

The authors declare that there are no conflicts of interest related to this article.

\section{References}

1. O. L. Landen, D. R. Farley, S. G. Glendinning, L. M. Logory, P. M. Bell, J. A. Koch, F. D. Lee, D. K. Bradley, D. H. Kalantar, C. A. Back, and R. E. Turner, Rev. Sci. Instrum. 72, 627 (2001).

2. L. C. Jarrott, B. Bachmann, T. Ma, L. R. Benedetti, F. E. Field, E. P. Hartouni, R. Hatarik, N. Izumi, S. F. Khan, O. L. Landen, S. R. Nagel, R. Nora, A. Pak, J. L. Peterson, M. B. Schneider, P. T. Springer, and P. K. Patel, Phys. Rev. Lett. 121, 085001 (2018).

3. A. G. Lynn and M. Gilmore, Rev. Sci. Instrum. 85, D609 (2014).

4. M. R. Gomez, S. A. Slutz, A. B. Sefkow, K. D. Hahn, S. B. Hansen, P. F. Knapp, P. F. Schmit, C. L. Ruiz, D. B. Sinars, E. C. Harding, C. A. Jennings, T. J. Awe, M. Geissel, D. C. Rovang, I. C. Smith, G. A. Chandler, G. W. Cooper, M. E. Cuneo, A. J. Harvey-Thompson, M. C. Herrmann, M. H. Hess, D. C. Lamppa, M. R. Martin, R. D. McBride, K. J. Peterson, J. L. Porter, G. A. Rochau, M. E. Savage, D. G. Schroen, W. A. Stygar, and R. A. Vesey, Phys. Plasmas 22, 056306 (2015).

5. S. V. Lebedev, F. N. Beg, S. N. Bland, J. P. Chittenden, A. E. Dangor, M. G. Harines, M. Zakaullah, S. A. Pikuz, T. A. Shelkovenko, and D. A. Hammer, Rev. Sci. Instrum. 72, 671 (2001).
6. Q. G. Yang, D. B. Liu, J. Mu, X. B. Huang, J. K. Dan, X. D. Xie, W. Deng, S. P. Feng, M. Wang, Y. Ye, Q. X. Peng, and Z. R. Li, Rev. Sci. Instrum. 87, 093706 (2016).

7. T. Ao, E. C. Harding, J. E. Bailey, R. W. Lemke, M. P. Desjarlais, S. B. Hansen, I. C. Smith, M. Geissel, A. Maurer, J. Reneker, D. Romero, D. B. Sinars, G. A. Rochau, and J. F. Benage, High Energy Density Phys. 18, 26 (2016).

8. R. S. Crum, M. A. Homel, D. C. Pagan, E. B. Herbold, D. Miller, J. Lind, B. J. Jensen, A. J. Iverson and M. C. Akin, J. Appl. Phys. 125, 025902 (2019).

9. J. Xiong, J. Q. Jia, G. Jia, R. R. Wang, W. Wang, S. Z. Fu, and W. D. Zheng, Chin. Phys. B 22, 065201 (2013).

10. S. Ohira, S. Fujioka, A. Sunahara, T. Johzaki, H. Nagatomo, S. Matsuo, N. Morio, J. Kawanaka, Y. Nakata, N. Miyanaga, H. Shiraga, H. Nishimura, and H. Azechi, J. Appl. Phys. 112 , 063301 (2012).

11. B. Loupias, F. Perez, A. B. mounaix, N. Ozaki, M. Rabec, L. E. Gloahec, T. A. Pikuz, A. Y. Faenov, Y. Aglitskiy, and M. Koenig, Laser Part. Beams 27, 601 (2009).

12. C. McGuffey, M. Dozieres, J. Kim, A. Savin, J. Park, J. Emig, C. Brabetz, L. Carlson, R. F. Heeter, H. S. McLean, J. Moody, M. B. Schneider, M. S. Wei, and F. N. Beg, Rev. Sci. Instrum. 89, 10F122 (2018).

13. D. K. Bradley, O. L. Landen, A. B. Bullocks, S. G. Glendnning, and R. E, Turner, Opt. Lett. 27, 134 (2002).

14. G. A. Kyrala, R. D. Fulton, E. K. Wahlin, L. A. Jones, G. T. Schappert, J. A. Cobble, and A. J. Taylor, Appl. Phys. Lett. 60, 2195 (1992).

15. P. K. Rambo, I. C. Smith, J. L. Porter Jr., M. J. Hurst, C. S. Speas, R. G. Adams, A. J. Garcia, E. Dawson, B. D. Thurston, C. Wakefield, J. W. Kellogg, M. J. Slattery, H. C. Ives III, R. S. Broyles, J. A. Caird, A. C. Erlandson, J. E. Murray, W. C. Behrendt, N. D. Neilsen, and J. M. Narduzzi, App. Opt. 44, 2421 (2005).

16. M. Koenig, A. Benuzzi-Mounaix, N. Ozaki, A. Ravasio, T. Vinci, S. Lepape, K. Tanaka, and D. Riley, AIP Conf. Proc. 845, 1421 (2006).

17. M. S. Schollmeier, P. F. Knapp, D. J. Ampleford, E. C. Harding, C. A. Jennings, D. C. Lamppa, G. P. Loisel, M. R. Martin, G. K. Robertson, J. E. Shores, I. C. Smith, C. S. Speas, M. R. Weis, J. L. Porter, and R. D. McBride, Rev. Sci. Instrum. 88, 103503 (2017).

18. P. Rambo, J. Schwarz, M. Schollmeier, M. Geissel, I. Smith, M. Kimmel, C. Speas, J. Shores, D. Armstrong, J. Bellum, E. Field, D. Kletecka, and J. Porter, Proc. SPIE 10014, 100140Z (2016).

19. M. J. Norman, J. E. Andrew, T. H. Bett, R. K. Clifford, J. E. England, N. W. Hopps, K. W. Parker, K. Porter, and M. Stevenson, Appl. Opt. 41, 3497 (2002).

20. C. A. Haynam, P. J. Wegner, J. M. Auerbach, M. W. Bowers, S. N. Dixit, G. V. Erbert, G. M. Heestand, M. A. Henesian, M. R. Hermann, K. S. Jancaitis, K. R. Manes, C. D. Marshall, N. C. Mehta, J. Menapace, E. Moses, J. R. Murray, M. C. Nostrand, C. D. Orth, R. Patterson, R. A. Sacks, M. J. Shaw, M. Spaeth, S. B. Sutton, W. H. Williams, C. C. Widmayer, R. K. White, S. T. Yang, and B. M. Van Wonterghem, Appl. Opt. 46, 3276 (2007).

21. J. P. Zou, A. M. Sautivet, J. Fils, L. Martin, K. Abdeli, C. Sauteret, and B. Wattellier, Appl. Opt. 47, 704 (2008)

22. C. C. Widmayer, J. M. Auerbach, R. B. Ehrlich, M. A. Henesian, J. T. Hunt, J. K. Lawson, D. Milam, P. A. Renard, D. R. Speck, P. J. Wegner, T. L. Weiland, W. H. Williams, C. R. Wolfe, and B. M. Van Wonterghem, Fusion Technol. 30, 464 (1996).

23. P. J. Wegner, M. A. Henesian, J. T. Salmon, L. G. Seppala, T. L. Weiland, W. H. Williams, and B. M. Van Wonterghem, Proc. SPIE 3492, 1019 (1999). 
24. X. Yu, L. Z. Dong, B. H. Lai, P. Yang, S. Q. Chen, W. J. Liu, S. Wang, G. M. Tang, J. S. Qiu, Z. J. Kang, Y. L. Liu, H. Liu, Y. Liu, Z. W. Fan, and B. Xu, Opt. Lett. 42, 2730 (2017).

25. R. A. Zacharias, N. R. Beer, E. S. Bliss, S. C. Burkhart, S. J. Cohen, S. B. Sutton, R. L. Van Atta, S. E. Winters, J. T. Salmon, M. R. Latta, C. J. Stolz, D. C. Pigg, and T. J. Arnold, Opt. Eng. 43, 2873 (2004).

26. S. Mainguy, C. Grosset-Grange, and E. Bordenave, J. Phys. Conf. Ser. 244, 032021 (2010).

27. D. E. Wang, D. X. Hu, Q. Yuan, Q. Xue, W. Zhou, Y. Yang, X. Zhang, X. W. Deng, Y. C. Wang, J. P. Zhao, W. Deng, X. F. Wei, W. J. Dai, J. Jing, Q. H. Zhu, and W. G. Zheng, Opt. Commun. 394, 92 (2017).

28. D. E. Wang, D. X. Hu, W. Zhou, X. W. Deng, Y. C. Wang, W. Deng, Q. Yuan, X. Zhang, L. Huang, T. H. Li, Q. Xue, H. Y. Yuan, Z. T. Peng, W. J. Dai, Q. H. Zhu, and F. Jing, Laser Phys. Lett. 10, 5002 (2013).

29. J. Heebner, M. Borden, P. Miller, S. Hunter, K. Christensen, M. Scanlan, C. Haynam, P. Wegner, M. Hermann, G. Brunton, E. Tse, A. Awwal, N. Wong, L. Seppala, M. Franks, E. Marley, K. Williams, T. Budge, M. Henesian, C. Stolz, T. Suratwala, M. Monticelli, D. Walmer, S. Dixit, C. Widmayer, J. Wolfe, J. Bude, K. McCarty, and J. M. DiNicola, Proc. SPIE 7916, 79160H (2011).
30. M. Barczys, S. W. Bahk, M. Spilatro, D. Coppenbarger, E. Hill, T. H. Hinterman, R. W. Kidder, J. Puth, T. Touris, and J. D. Zuegel, Proc. SPIE 8602, 86020F (2013).

31. C. A. Haynam, P. J. Wegner, J. M. Auerbach, M. W. Bowers, S. N. Dixit, G. V. Erbert, G. M. Heestand, M. A. Henesian, M. R. Hermann, K. S. Jancaitis, K. R. Manes, C. D. Marshall, N. C. Mehta, J. Menapace, E. Moses, J. R. Murray, M. C. Nostrand, C. D. Orth, R. Patterson, R. A. Sacks, M. J. Shaw, M. Spaeth, S. B. Sutton, W. H. Williams, C. C. Widmayer, R. K. White, S. T. Yang, and B. M. Van Wonterghem, Appl. Opt. 46, 3276 (2007).

32. M. L. Spaeth, K. R. Manes, C. C. Widmayer, W. H. Williams, P. K. Whitman, M. A. Henesian, I. F. Stowers, and J. Honig, Opt. Eng. 43, 2854 (2004).

33. M. Tabatabaian, COMSOL for Engineers (Mercury Learning \& Information, 2014).

34. R. W. Pryor, Multiphysics Modeling Using COMSOL: A First Principles Approach (Jones and Bartlett Publishers, 2011).

35. E. J. Fernández and P. Artal, Opt. Express 11, 1056 (2003).

36. M. Booth, T. Wilson, H. B. Sun, T. Ota, and S. Kawata, Appl. Opt. 44, 5131 (2005).

37. P. M. Celliers, K. G. Estabrook, R. J. Wallace, J. E. Murray, L. B. Da Silva, B. J. MacGowan, B. M. Van Wonterghem, and K. R. Manes, Appl. Opt. 37, 2371 (1998).

38. J. E. Murray, D. Milam, C. D. Boley, K. G. Estabrook, and J. A. Caird, Appl. Opt. 39, 1405 (2000). 